\title{
Effets génétiques sur les caractères des portées issues de trois souches de lapins utilisées en croisement
}

\author{
J.M. BRUN et R. ROUVIER \\ I.N.R.A., Station d'Amélioration génétique des Animaux \\ Centre de Recherches de Toulouse, BP 12, F 31320 Castanet-Tolosan
}

\begin{abstract}
Résumé
A partir d'une expérience de croisement à double étage entre les souches de lapins Californienne $(C A)$, Néo-Zélandaise Blanche (NZ) et Petite Russe (PR) sont estimés leurs effets génétiques directs, maternels, grand-maternels, les hétérosis directs et maternels, sur les caractères de la portée (taille à la naissance et au sevrage ; poids au sevrage). L'effet maternel varie largement entre les souches $C A, N Z$ et $P R$ pour le nombre de nés vivants par portée $\left(+0,62,+0,08\right.$ et $-0,70^{*}$ respectivement) et le nombre de sevrés par portée sevrée $\left(+1,20^{* *},-0,26\right.$ et $-0,94^{* *}$ respectivement). Il traduit, pour le premier caractère, les valeurs additives des souches pour le taux d'ovulation. L'opposition entre effets maternels favorables (ovulation, viabilité naissance-sevrage) et grand-maternels défavorables (viabilité) de la Californienne en fait une souche paternelle de la lapine métisse. La Néo-Zélandaise Blanche complémente la Californienne par son effet grand-maternel favorable (lié à son aptitude laitière) sur la taille et le poids de portées au sevrage et confère à la femelle $\left(\sigma^{\prime} C A \times q N Z\right)$ une supériorité de 18 p. 100 par rapport à la moyenne. La souche Petite Russe, pénalisée par son faible niveau d'ovulation et sa petite taille, a un effet direct favorable sur la viabilité et conduit à des hétérosis spécifiques avec les deux autres. Ainsi, les portées de lapines métisses $\left(O^{\prime} C A \times \bigcirc P R\right)$ et $\left(O^{\prime} P R \times \bigcirc N Z\right)$ cumulent des effets génétiques additifs et non-additifs favorables. Le gain total par hétérosis sur le nombre et le poids de lapereaux sevrés est de 22 p. 100. Les conséquences de ces résultats sur les méthodes d'amélioration des croisements sont envisagées.
\end{abstract}

Mots clés : Lapin, effets génétiques directs, effets génétiques maternels, hétérosis, caractères de la portée.

\section{Summary}

\section{Genetic effects on litter traits among three strain crosses in rabbit}

Direct, maternal and grand-maternal genetic effects along with direct and maternal heterosis on litter traits (size at birth and weaning, weaning weight), are estimated from a crossbreeding experiment using Californian (CA), New-Zealand White (NZ) and Small Russian (SR) rabbit strains. The amount of variation between $C A, N Z$ and $S R$ strains is particularly important for maternal effects, either on litter size at birth $\left(+0,62,+0,08\right.$ and $-0,70^{*}$ respectively) or at weaning $\left(+1,20^{* *},-0,26\right.$ and $-0,94^{* *}$ respectively). Maternal effects on litter size at birth reflect the additive genetic values of the strains for ovulation rate. The opposition between favourable maternal effects (ovulation, viability from birth to weaning) and unfavourable grandmaternal effects (viability) of the $C A$ strain makes it suitable for the paternal strain of crossbred does. The $\left(\sigma^{\prime} C A \times Q N Z\right)$ doe litters capitalize favourable maternal effects transmitted by $C A$ 
grand-father and favourable grand-maternal effects (related to milking ability $N Z$ female. This complementarity increases weaning trait performance by 18 per 100 . The $S R$ is penalized by its low ovulation rate and small size but shows a favourable direct effect on viability and leads to specific heterosis. Thus, litters from $\left(\delta^{\prime} C A \times \bigcirc S R\right)$ and $\left(\zeta^{\prime} S R \times Q N Z\right)$ crossbred does accumulate some additive and non-additive favourable genetic effects. Total gain from heterosis on the number weaned and litter weight at weaning averages 22 per 100 . The implications of these results for selection methods to improve the crossbreds are discussed.

Key words : Rabbit, litter traits, direct genetic effects, maternal genectic offects, heterosis.

\section{Introduction}

La rentabilité d'un élevage de production de lapins pour la boucherie dépend d'abord du nombre et du poids de lapereaux sevrés par cage de mère et par an. Deux facteurs zootechniques principaux contribuent à accroître cette production : le nombre de portées sevrées par cage de mère et par an qui dépend de la technique d'élevage, et la productivité numérique et pondérale par portée sevrée qui dépendrait plus des animaux. Ainsi, dans le but de choisir une lapine métisse à forte productivité numérique et pondérale par portée sevrée, une expérience de croisement à double étage entre les souches Californienne, Néo-Zélandaise Blanche et Petite Russe a été réalisée en 1970-1972, dans l'élevage expérimental de l'I.N.R.A. à Toulouse. De premiers travaux sur les résultats de cette expérience ont estimé l'hétérosis pour quelques caractères de reproduction chez le lapin (MATHERON \& PoujARDIEU, 1976) et étudié leur variation génétique en termes d'effets globaux des souches en tant que parents ou grand-parents des portées (MATHERON \& Rouvier, 1978). MATHERON \& MAULÉon (1979) ont défini ces caractères comme attributs de la portée et non de la mère et montré qu'ils dépendent de l'action conjointe de groupes de gènes différents chez les lapereaux, leur mère, leur grand-mère. Le modèle de DiCKERSON (1969) peut être utilisé pour estimer les effets de ces groupes de gènes sur le plan statistique : effets génétiques direct, maternel, grand-maternel et d'hétérosis. L'objectif de la présente étude est d'estimer tous ces effets génétiques sur les caractères des portées issues de cette expérience de croisement et d'en discuter les mécanismes d'action. Cela permettra d'accroître les connaissances génétiques et biologiques de ces 3 souches et d'étudier l'orientation de leur sélection.

\section{Matériel et méthodes}

\section{A. Modèle biologique et protocole expérimental}

Trois souches fermées depuis 1968 sont utilisées : Californienne (issue d'animaux des races Californienne et Grande Russe), Néo-Zélandaise Blanche, et Petite Russe. Les 2 premières sont de taille adulte moyenne $(4 \mathrm{~kg})$, la dernière de petite taille $(2,5 \mathrm{~kg})$. Dans une $1^{\text {re }}$ génération de croisement, mise en place en septembre 1970 et d'une durée d'un an, 108 lapines des 3 souches sont accouplées avec des mâles des mêmes souches suivant un plan factoriel $3 \times 3$. L'analyse porte sur les performances de 730 mises bas. Dans une $2^{\mathrm{e}}$ génération, mise en place en octobre 1971 et d'une durée d'un an, 18 lapines issues de chacun des 9 types d'accouplements de $11^{\text {re }}$ génération sont mises en reproduction avec des mâles d'une souche Fauve de Bourgogne. L'analyse porte sur les performances de 153 femelles ayant donné 546 mises bas. 
Les lapines étaient présentées pour la première fois au mâle à l'âge d'environ 5 mois, ainsi que le $10^{\mathrm{e}} \mathrm{j}$ après chaque mise bas ; les lapereaux étaient séparés de leur mère (sevrage) à l'âge de $30 \mathrm{j}$. Les femelles étaient élevées en cages grillagées individuelles, avec boîte à nid métallique, dans un bâtiment conventionnel propre, muni de Senêtres et chauffé en hiver de façon à maintenir une température ambiante d'environ $18{ }^{\circ} \mathrm{C}$. La durée d'éclairement était de $16 \mathrm{~h}$ par $24 \mathrm{~h}$. Les femelles étaient nourries avec un aliment concentré complet du commerce. Les jeunes avaient accès à la trémie à granulés de leur mère. Les lapines allaitantes étaient nourries ad libitum, les mâles et lapines non gestantes recevant une ration de $130 \mathrm{~g}$ par jour. Les reproducteurs étaient distribués dans les cages du bâtiment d'élevage de façon à éviter une confusion entre un effet type génétique et un effet localisation. Aucun traitement médical n'a été effectué sur les animaux.

\section{B. Méthode d'estimation des effets génétiques}

Les caractères analysés sont le nombre de lapereaux nés vivants par mise bas, le nombre de lapereaux sevrés par portée sevrée, le poids total de portée sevrée et le poids individuel moyen par portée des lapereaux sevrés. Ces variables sont considérées comme des caractères de la portée.

Suivant Foulley (1983, comm. pers.), nous avons établi, pour chacune des 2 générations, le tableau de décomposition des valeurs génotypiques moyennes des 9 types génétiques en fonction des effets génétiques de Dickerson (1969) que l'on veut estimer (tableaux 1 et 2). Assortis d'un reparamétrage, ces tableaux définissent un système d'équations de plein rang. Les cöefficients définissant les effets génétiques à partir des valeurs génotypiques moyennes s'obtiennent par inversion matricielle. Les tableaux 3 et 4 donnent la partie de ces matrices inverses correspondant aux effets génétiques à estimer. Ceux-ci sont les paramètres $\mu_{1}$ et $\mu_{2}$ respectivement pour les générations 1 et 2 ; les effets génétiques directs $\left(\mathrm{g}_{\mathrm{i}}^{\mathrm{I}}\right)$, maternels $\left(\mathrm{g}_{\mathrm{i}}^{\mathrm{M}}\right)$ et grand-maternels $\left(\mathrm{g}_{\mathrm{i}}^{\mathrm{M}}\right)$ des 3 souches $\mathrm{i}(\mathrm{i}=C A, N Z$ ou $P R)$; les hétérosis directs $\left(\mathrm{h}_{\mathrm{ij}}^{\mathrm{L}}\right)$ et maternels $\left(\mathrm{h}_{\mathrm{i}}^{\mathrm{M}}\right)$ relatifs aux couples de souches $i$ et $j$, ainsi que les effets de réciprocité $r_{1}$ et $r_{2}$ respectivement pour les générations 1 et 2 , supposés les mêmes pour chaque couple de croisements réciproques. En $1^{\text {re }}$ génération, les 9 croisements permettent d'estimer 9 paramètres linéairement indépendants tels que $\mu_{1}, g_{C_{A}}-g_{N Z}^{I}, g_{p_{R}}-g_{N Z}^{I}\left(g_{C A}^{M}\right.$ $\left.+g_{C A}^{M}\right)-\left(g_{N Z}^{M}+g_{N Z}^{\prime}\right), \quad\left(g_{P R}^{M}+g_{P R}^{M \prime}\right)-\left(g_{N Z}^{M}+g_{N Z}^{\prime}\right), \quad h_{C A}^{L} \times N Z, \quad h_{C A}^{\prime} \times P R, \quad h_{N Z \times P R}^{L}$, $r_{1}$. En $2^{e}$ génération, l'analyse exploite les résultats de 9 croisements auxquels on ajoute 2 estimées de l'expérience précédente. Il y a donc 11 paramètres estimables linéairement indépendants, soit par exemple $\mu_{2}, 2$ différences d'effets directs, 2 différences d'effets maternels, 2 différences d'effets grand-maternels, 3 effets d'hétérosis maternels et 1 effet de réciprocité. Les contraintes introduites $\sum_{i} g_{i}^{I}=0$ et $\underset{i}{\sum}\left(g_{i}^{M}+g_{i}^{M^{\prime}}\right)=0$ en $1^{\text {re }}$ génération et $\sum_{i} g_{1}^{I}=0 ; \sum_{i} g_{i}^{M}=0$ et $\sum_{i} g_{i}^{M^{\prime}}=0$ en $2^{\text {e }}$ génération, ne sont là que pour rendre calculables les paramètres surnuméraires. Cette méthode permet d'étendre le modèle statistique de EISEN et al. (1983) à l'interprétation de 2 générations successives de croisements.

En $2^{\mathrm{e}}$ génération, les $\mathrm{g}_{1}^{\mathrm{I}}(\mathrm{i}=C A, N Z$ ou $P R)$ sont indissociables des hétérosis individuels $h_{i t}^{h}$ où $t=F B$, car ils sont affectés de coefficients proportionnels. En conséquence, on retient pour $\mathrm{g} \mid$ les estimées issues de la première génération. 


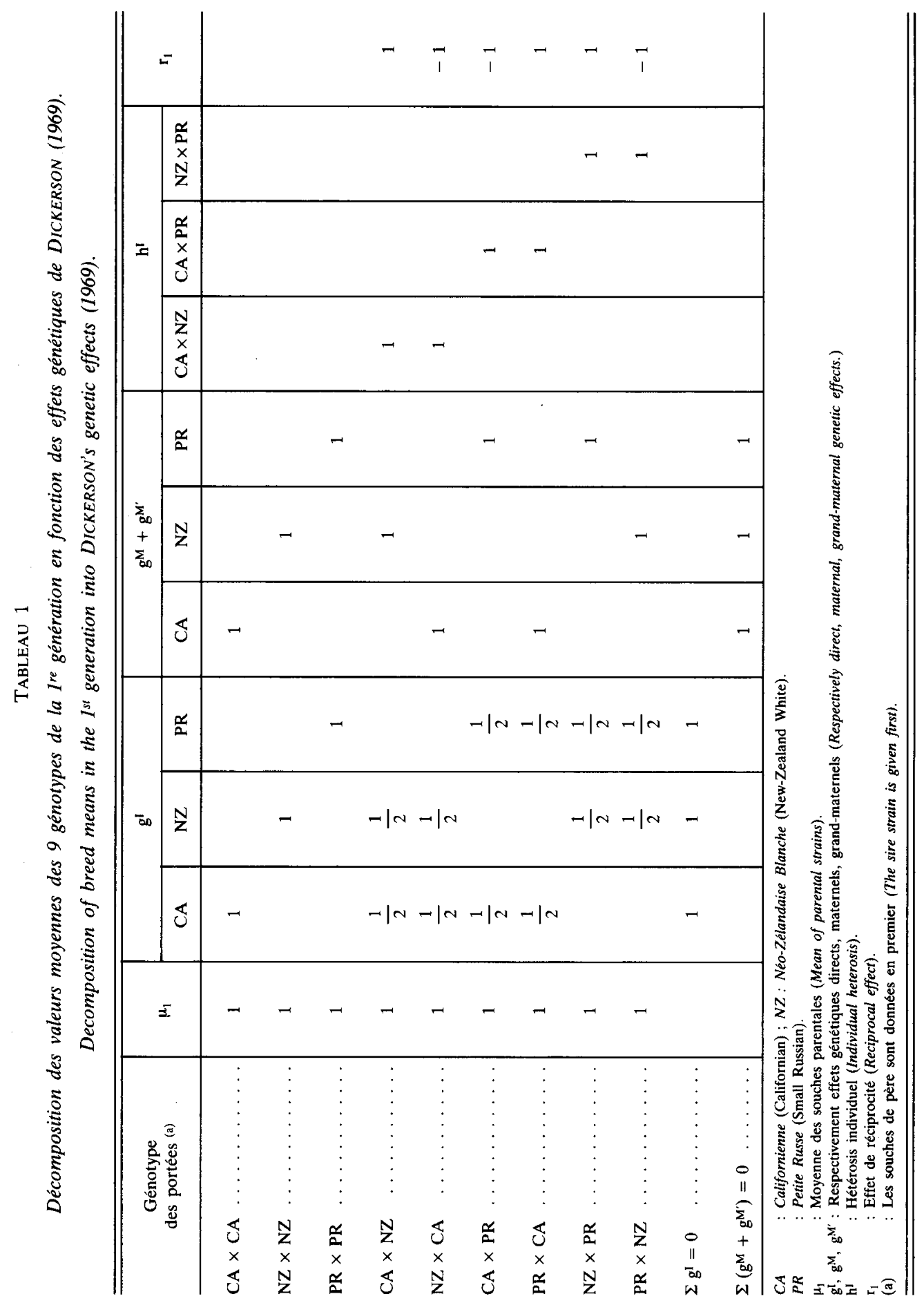


EFFETS GÉNÉTIQUES EN CROISEMENT

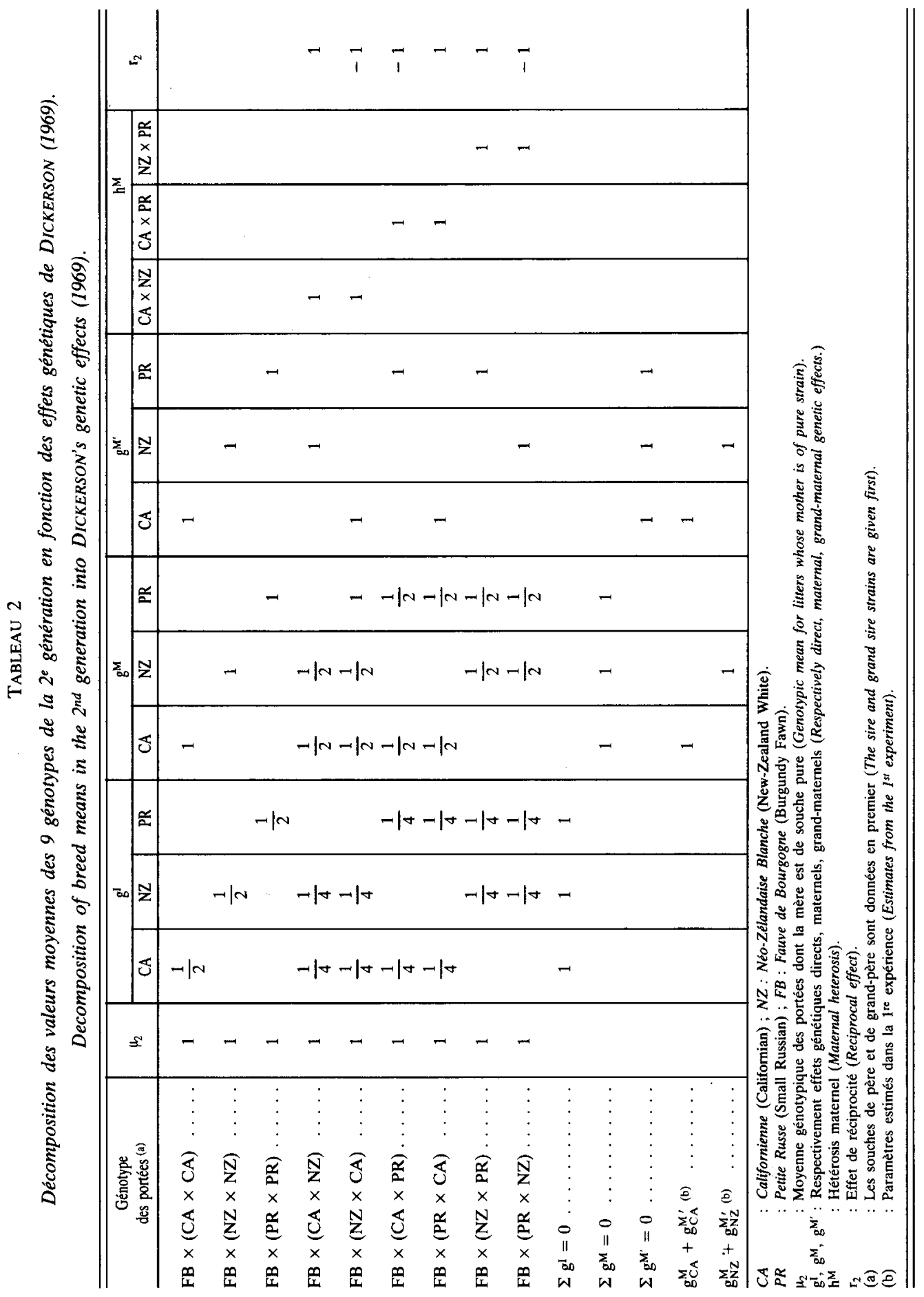




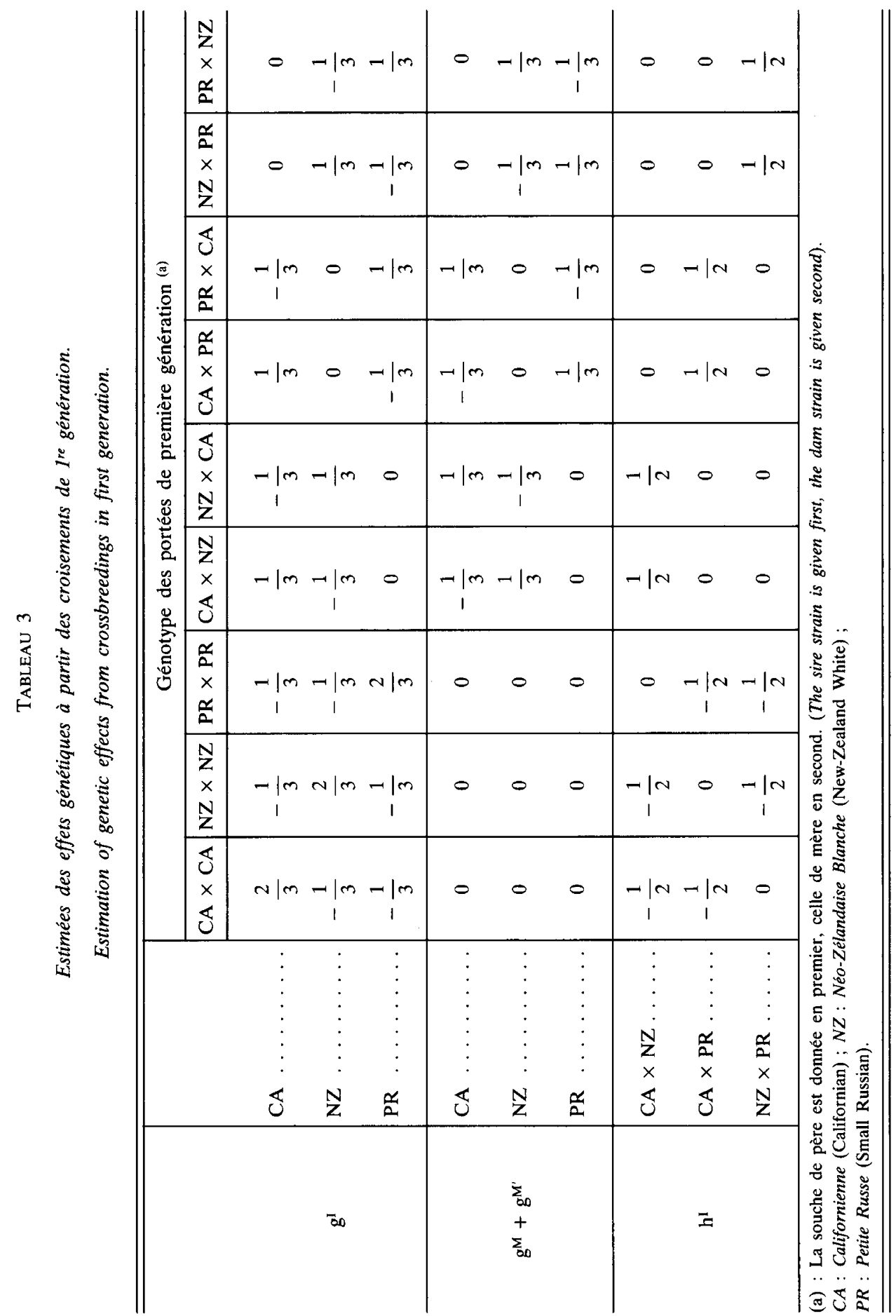




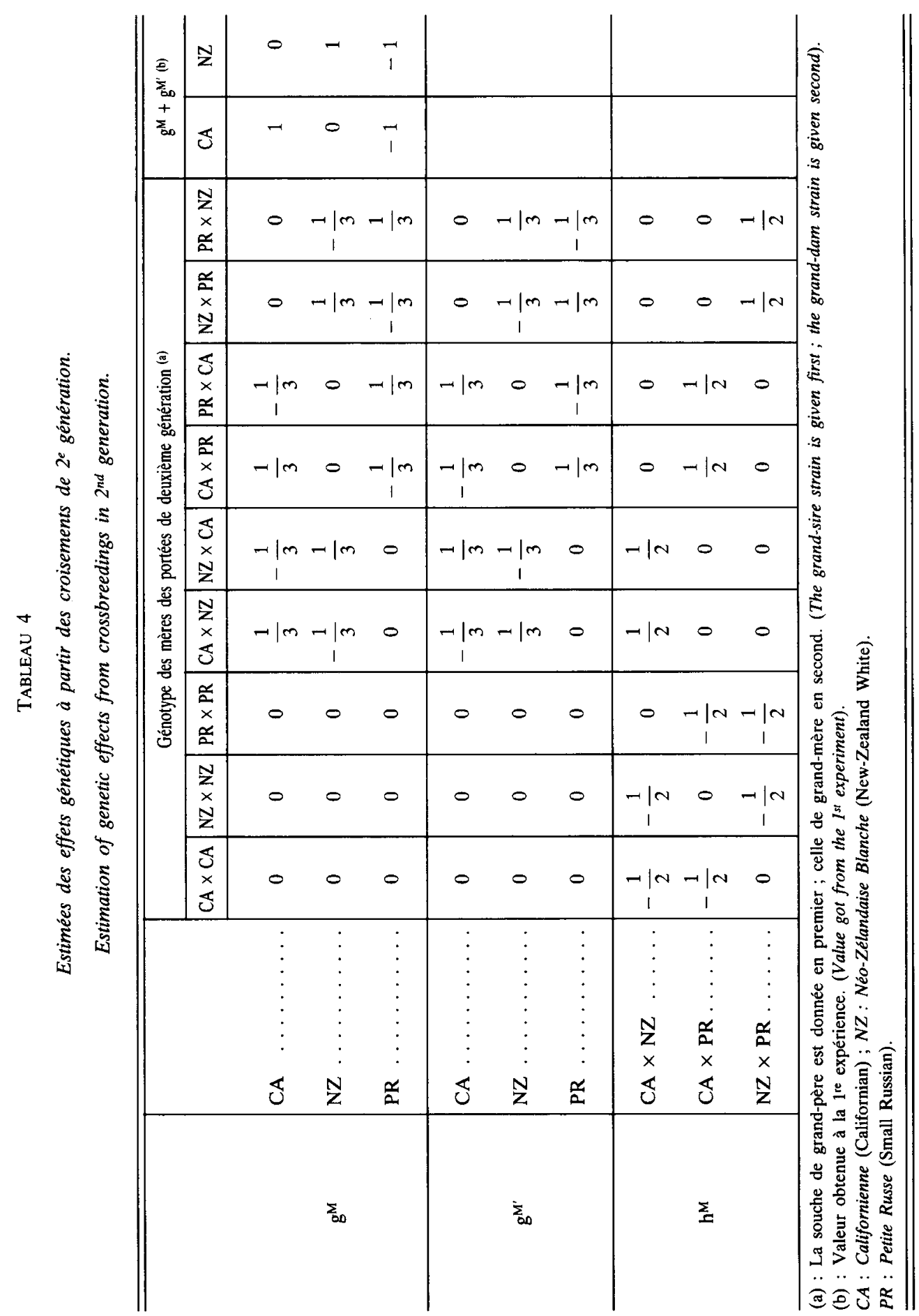


Les valeurs génotypiques moyennes ont été estimées par la méthode des moindres carrés suivant le modèle :

$$
y_{i j k l}=\mu+\alpha_{i}+g_{j k}+e_{i j k l}
$$

ou $y_{i j k l}$ est la lième observation, de numéro de portée $i$, de race de père $j$ (ou de grand-père dans le cas de la $2^{\mathrm{e}}$ génération) et de race de mère $\mathrm{k}$ (ou de grand-mère dans le cas de la $2^{\mathrm{e}}$ génération).

$\mu$ est la moyenne générale de la génération.

$\alpha_{i}$ est l'effet numéro de portée (i varie de 1 à 7 ). génération.

$\mathrm{g}_{\mathrm{jk}}$ est l'effet de génotype $(\mathrm{j} \times \mathrm{k})$ de la portée (ou de la mère) en $1^{\text {re }}\left(\right.$ ou en $2^{\mathrm{e}}$ )

$\mu+\mathrm{g}_{\mathrm{jk}}$ est donc la valeur génotypique du croisement $\mathrm{jk}$ ( $\mathrm{j}$ et $\mathrm{k}$ varient de $1 \mathrm{a} 3$ ).

$e_{i j k l}$ est une variable aléatoire de variance $\sigma^{2} e$.

Une analyse préliminaire a permis de tester l'absence d'interaction significative entre effets numéro de portée et type génétique. Les effets génétiques s'expriment donc sous la forme de combinaisons linéaires des estimées des valeurs génotypiques moyennes de chaque type de croisement. Pour les effets d'hétérosis $\left(\mathrm{h}^{\mathrm{I}}, \mathrm{h}^{\mathrm{M}}\right)$ cela revient à les estimer suivant la définition classique de différence entre les moyennes des 2 croisés réciproques et des 2 souches parentales. La signification des effets génétiques par rapport à zéro a été testée, après calcul des variances des combinaisons linéaires, à partir des variances des estimées du modèle (1), par un test de Student sous l'hypothèse de normalité des distributions et d'indépendance d'échantillonnage des géniteurs mâles et femelles d'une génération donnée.

\section{Résultats}

\section{A. Résultats globaux}

Les tableaux 5 et 6 donnent, pour les générations 1 et 2 respectivement, les moyennes et variances résiduelles des différents caractères analysés, les estimées des valeurs moyennes des différents génotypes ainsi que les effets moyens des souches comme parents ou grands-parents des portées. On peut noter une réduction de la variabilité résiduelle des tailles de portées, mais non des poids, entre la $1^{\text {re }}$ et la $2^{\mathrm{e}}$ génération. Les portées ayant une mère Petite Russe ou même seulement un grand-père maternel de cette souche, ont une taille (à la naissance) et un poids au sevrage significativement inférieurs à la moyenne. Quand la mère, ou seulement le grand-père, est de souche Californienne, on observe au contraire les plus fortes prolificité et productivité pondérale. La souche Néo-Zélandaise Blanche excelle également sur les tailles et poids de portées au sevrage, mais seulement en temps que souche grand-maternelle.

\section{B. Effets génétiques additifs et d'hétérosis}

Le tableau 7 indique les valeurs et les significations des effets génétiques directs, maternels et grand-maternels pour les différents caractères. 


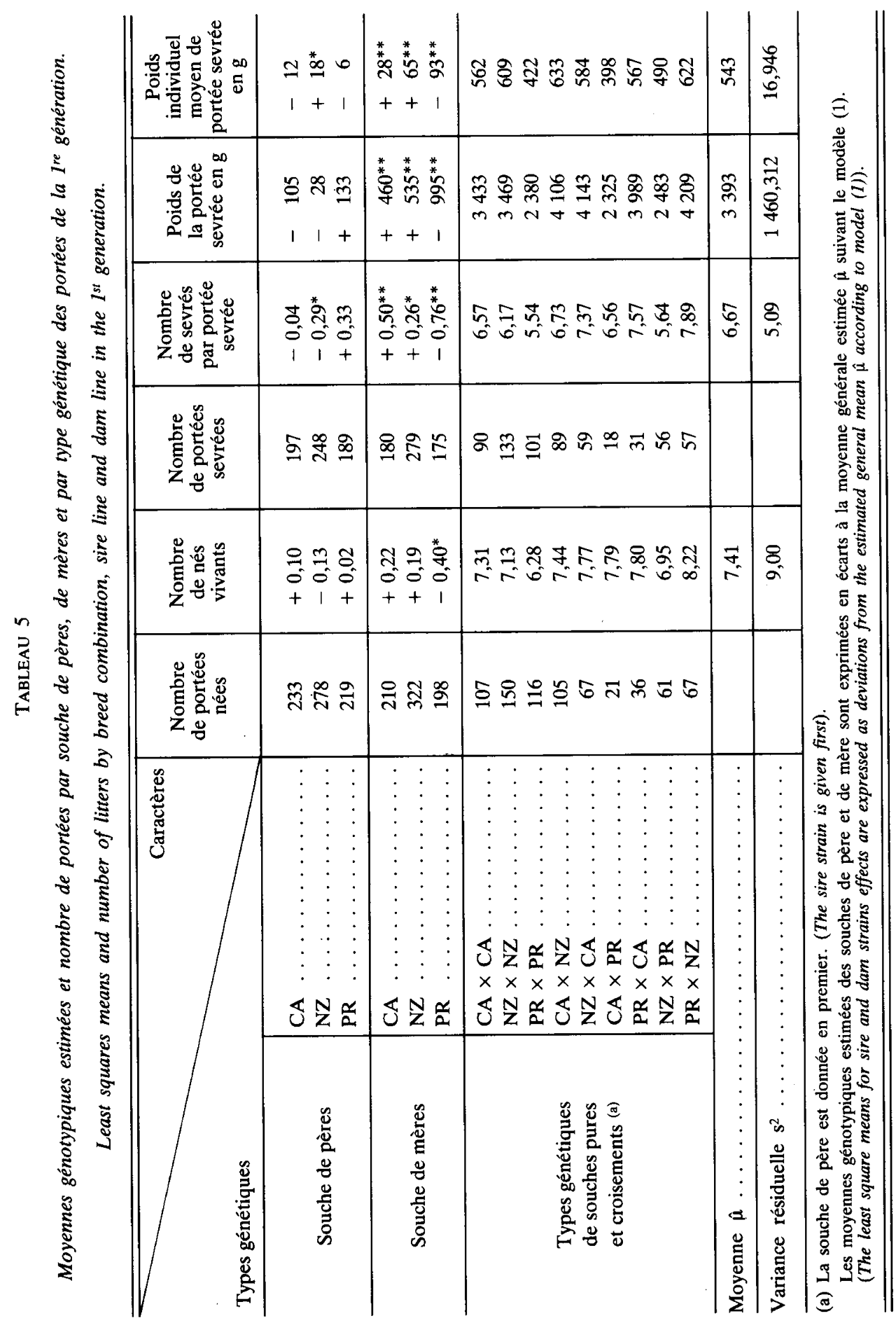




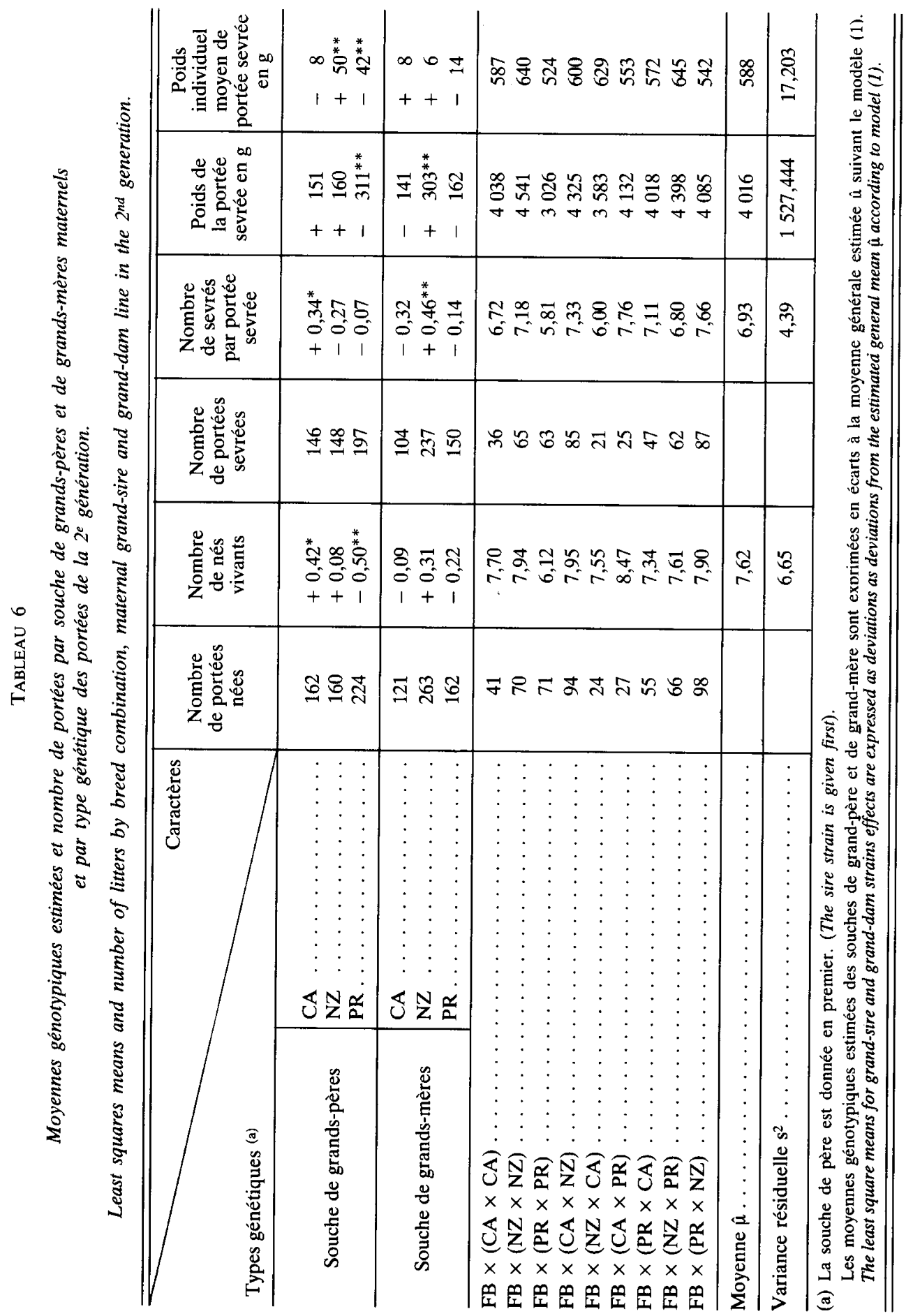




\section{TABLEAU 7}

Estimées des effets génétiques additifs directs, maternels et grand-maternels, sur les caractères de la portée, pour chacune des 3 souches $\mathrm{CA}, \mathrm{NZ}, \mathrm{PR}$, obtenues à partir des 2 générations successives de croisement.

Estimated additive genetic effects (direct, maternal, grand-maternal) for the litter traits, between the 3 strains $\mathrm{CA}, \mathrm{NZ}, \mathrm{PR}$, calculated from the 2 generations of crossbreeding.

\begin{tabular}{|c|c|c|c|c|c|c|}
\hline \multirow{2}{*}{$\frac{\text { Caractères }}{\text { Effets }}$} & \multicolumn{3}{|c|}{ Nés vivants par portée } & \multicolumn{3}{|c|}{ Sevrés par portée } \\
\hline & $\mathrm{g}^{\mathrm{I}}$ & $\mathrm{g}^{\mathrm{M}}$ & $\mathrm{g}^{\mathrm{M}^{\prime}}$ & gl & $\mathrm{g}^{\mathrm{M}}$ & $\mathrm{g}^{\mathrm{M}^{\prime}}$ \\
\hline Souches : & & & & & & \\
\hline CA $\ldots$ & $+0,29$ & $+0,62$ & $-0,51^{*}$ & $-0,07$ & $+1,20^{* *}$ & $-0,65^{* *}$ \\
\hline NZ & $-0,09$ & $+0,08$ & $+0,23$ & $-0,46^{*}$ & $-0,26$ & $+0,80^{* *}$ \\
\hline $\mathrm{PR} \ldots \ldots \ldots \ldots$ & $-0,20$ & $-0,70^{*}$ & $+0,28$ & $+0,53$ & $-0,94^{* *}$ & $-0,15$ \\
\hline
\end{tabular}

\begin{tabular}{|c|c|c|c|c|c|c|}
\hline \multirow{2}{*}{$\begin{array}{c}\text { Caractères } \\
\text { Effets }\end{array}$} & \multicolumn{3}{|c|}{ Poids total de portée sevrée en $\mathrm{g}$} & \multicolumn{3}{|c|}{$\begin{array}{l}\text { Poids individuel moyen } \\
\text { de portée sevrée en } \mathrm{g}\end{array}$} \\
\hline & $\mathrm{g}^{\mathbf{l}}$ & $\mathrm{g}^{\mathrm{M}}$ & $\mathrm{g}^{\mathbf{M}^{\prime}}$ & $g^{I}$ & $\mathrm{~g}^{\mathrm{M}}$ & $g^{M^{\prime}}$ \\
\hline Souches : & & & & & & \\
\hline CA & -228 & $+849^{* *}$ & $-282^{*}$ & -9 & +24 & +16 \\
\hline NZ & -188 & $+421^{* *}$ & +142 & $+18^{*}$ & $+104^{* *}$ & $-44^{* *}$ \\
\hline PR . & $+416^{* *}$ & $-1270^{* *}$ & +140 & -9 & $-128^{* *}$ & $+28^{*}$ \\
\hline
\end{tabular}

g : Effets génétiques directs (direct genetic effects).

$\mathrm{g}^{\mathrm{M}}$ : Effets génétiques maternels (maternal genetic effects).

$\mathrm{g}^{\mathrm{M}}$ : Effets génétiques grand-maternels (grand-maternal genetic effects).

* : Valeur significativement différente de 0 (significant value).

** : Valeur très significativement différente de 0 (highly significant value).

Pour le caractère nés vivants par mise bas, les effets directs ne sont pas significatifs. On note une opposition entre effets maternel et grand-maternel pour la Californienne, une position intermédiaire de la Néo-Zélandaise Blanche pour ces 2 effets, une valeur négative significative de l'effet maternel de la Petite Russe.

Pour le caractère nombre de sevrés par portée sevrée, il apparaît des effets directs significatifs qui ne l'étaient pas à la naissance. On note l'amplitude de la variation, importante par rapport à la valeur moyenne du caractère, due aux effets maternels et dans une moindre mesure aux effets grand-maternels. Les écarts de 2,14 et 1,45 lapereaux sevrés entre extrêmes pour les effets maternels et grand-maternels représentent respectivement de l'ordre de 30 et 20 p. 100 de la moyenne du caractère. On note une opposition entre effets direct et maternel pour la Petite Russe, entre effets maternel et grand-maternel pour la souche Californienne.

Pour le caractère poids total de portée sevrée, les effets maternels se rangent comme pour le nombre de sevrés par portée sevrée. Ils sont respectivement pour les 
3 souches de $+849^{* *},+421^{* *},-1270^{* *}$ g. L'amplitude de variation due aux effets grand-maternels est faible, seul l'effet de la souche Californienne étant significatif $\left(-282^{*} \mathrm{~g}\right)$.

Pour le caractère poids individuel moyen par portée de lapereaux sevrés, l'effet direct de la Néo-Zélandaise Blanche est significatif $\left(+18^{*} \mathrm{~g}\right)$, les effets maternels ont les valeurs extrêmes pour la Néo-Zélandaise Blanche et la Petite Russe $\left(+104^{* *} \mathrm{~g}\right.$ et $-128^{* *} \mathrm{~g}$ respectivement), la Californienne étant intermédiaire. L'amplitude de la variation due aux effets grand-maternels est moins importante, mais ceux-ci sont significatifs pour la Néo-Zélandaise Blanche $\left(-44^{* *} \mathrm{~g}\right)$ et la Petite Russe $\left(+28^{*} \mathrm{~g}\right)$. On note aussi pour ces 2 souches une opposition entre effets maternel et grand-maternel pour le poids individuel moyen de portée sevrée.

La race Californienne présente les plus forts effets maternels pour les 3 caractéristiques importantes que sont le nombre de nés vivants $(+0,62)$, le nombre de sevrés par portée $\left(+1,20^{* *}\right)$ et le poids total de portée sevrée $\left(+849^{* *} \mathrm{~g}\right)$, la Néo-Zélandaise Blanche l'emportant pour le poids individuel moyen du lapereau sevré $\left(+104^{* *} \mathrm{~g}\right)$.

\section{Tableau 8}

$1^{r e}$ génération. Hétérosis sur les effets génétiques directs dans le croisement des 3 souches CA, NZ, PR, exprimé en pourcentage de la moyenne des souches parentales.

$1^{\text {st }}$ generation. Heterosis for direct effects in crossbreeding of the 3 strains CA, NZ, PR, expressed in percentage of the parental mean.

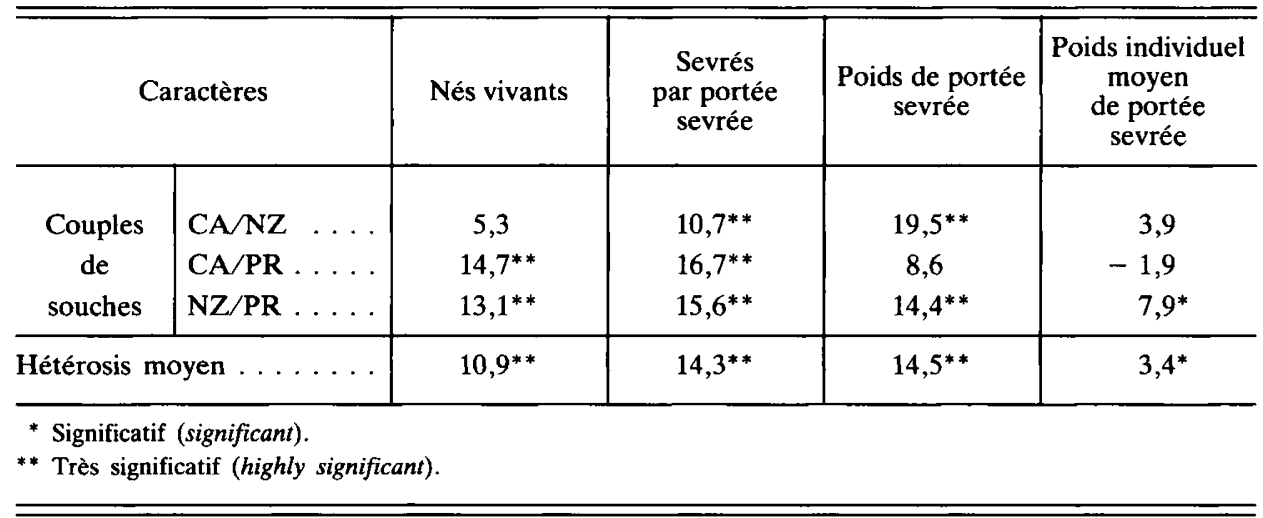

Les valeurs des effets d'hétérosis spécifiques et moyens sont indiquées en p. 100 aux tableaux 8 (hétérosis direct) et 9 (hétérosis maternel). Les hétérosis moyens sont plus importants sur les effets directs que sur les effets maternels et plus importants sur les tailles et poids de portées que sur les poids individuels moyens des lapereaux sevrés. Le croisement des souches Californienne et Néo-Zélandaise Blanche ne donne pas lieu à un hétérosis direct sur le nombre de nés vivants par mise bas ni sur le poids individuel moyen par portée des lapereaux sevrés, alors que celui-ci est significatif pour le nombre de lapereaux sevrés par portée et le poids de portée sevrée. Le croisement des souches Californienne et Petite Russe ne donne pas lieu à hétérosis direct significatif pour le poids de portée sevrée et le poids individuel moyen par portée des lapereaux sevrés. 


\section{TABleaU 9}

$2^{e}$ génération. Hétérosis sur les effets génétiques maternels dans le croisement des 3 souches $\mathrm{CA}, \mathrm{NZ}, \mathrm{PR}$, exprimé en pourcentage de la moyenne des souches parentales.

$2^{\text {nd }}$ generation. Heterosis for maternal genetic effects in crossbreeding of the 3 strains CA, NZ, PR, expressed in percentage of the parental mean.

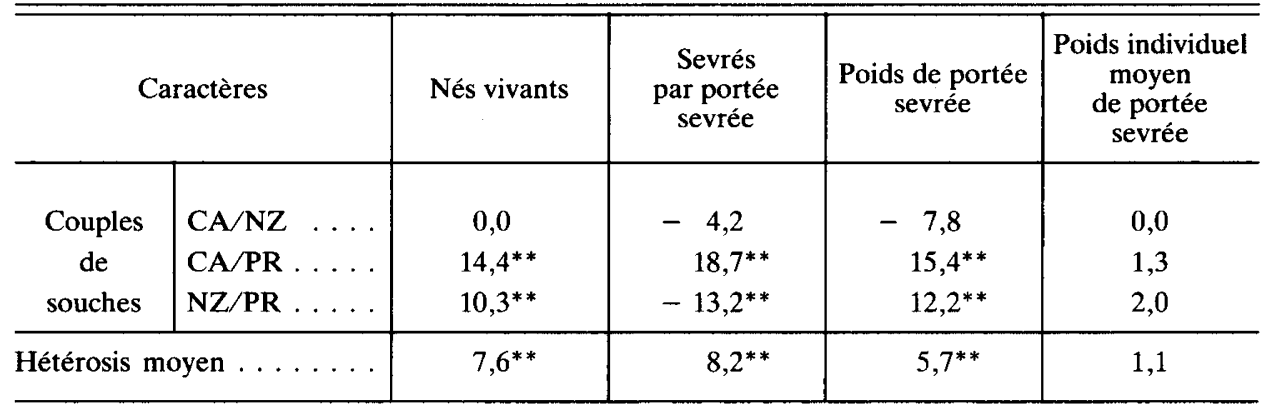

* Significatif (significant).

** Très significatif (highly significant).

Le croisement des souches Néo-Zélandaise Blanche et Petite Russe donne lieu à hétérosis sur les effets directs, significatifs pour tous les caractères étudiés. Les hétérosis maternels dépendent des couples de souches et sont donc spéficiques. La lapine métisse Californienne $\times$ Néo-Zélandaise Blanche et sa réciproque ne présentent pas d'hétérosis maternel, les 2 autres lapines métisses en présentent un, la lapine métisse Californienne $\times$ Petite Russe (et sa réciproque) présentent les valeurs les plus élevées d'hétérosis maternel.

\section{Discussion}

Alenda et al. (1980) ont développé des méthodes pour estimer les paramètres génétiques à partir des valeurs moyennes de croisements obtenus sur 2 générations, pour les poids à la naissance et au sevrage, des races Angus, Charolais, Hereford. La méthodologie utilisée ici est comparable. Notre discussion privilégiera l'interprétation des effets génétiques estimés en termes de mécanismes biologiques ou de déterminismes génétiques des caractères étudiés. Ces mécanismes biologiques ont pour base l'ovulation, la viabilité des œufs (pré et post-natale), l'aptitude laitière des lapines, la taille adulte des souches. Nous discuterons également les applications de cette étude au choix de la méthode pour améliorer la productivité numérique et pondérale au sevrage d'une lapine métisse.

\section{A. Effets génétiques directs, maternels et grand-maternels}

L'effectif de portée à la naissance est, avec la viabilité naissance-sevrage, une composante de l'effectif de portée sevrée. Les résultats de la présente analyse ne mettent pas en évidence, entre ces 3 souches, une variation due aux effets génétiques directs 
sur la viabilité des blastocystes, des embryons, et périnatale, qui se manifesterait par des effets directs sur le nombre de nés vivants, alors que Françoise Hulot \& MATHERON (1979) ont mis en évidence l'aptitude de la souche Petite Russe pour les effets génétiques directs sur la viabilité embryonnaire (à $16 \mathrm{j}$ de gestation). Les effectifs de portée à $16 \mathrm{j}$ de gestation et à la naissance seraient donc soumis à des déterminismes génétiques additifs directs différents. Par contre, les 3 souches Californienne, Néo-Zélandaise Blanche, Petite Russe se classent pour leurs effets maternels sur le nombre de nés vivants par mise bas comme pour leurs valeurs additives pour le taux d'ovulation estimées par ces auteurs à $0,69^{* *},-0,18$ et $-0,50^{*}$ ovules pondus. La valeur additive pour le taux d'ovulation est donc entre ces 3 souches une composante mesurable de leurs effets maternels sur le nombre de lapereaux nés vivants par mise bas. En l'absence de mise en évidence d'effet direct, ce résultat donnerait un moyen de mesure indirecte, par le nombre de nés vivants d'une portée d'une lapine métisse, de la valeur additive pour le taux d'ovulation de la souche grand-paternelle.

L'effet grand-maternel significativement défavorable chez la Californienne sur le nombre de nés vivants par mise bas, en opposition avec l'effet maternel de cette souche qui représenterait son niveau d'ovulation, pourrait traduire un effet grand-maternel défavorable sur la viabilité embryonnaire. Cela précise pour les effets génétiques en cause, les résultats de Françoise Hulot \& MATHERON (1981) qui indiquent que la Californienne se caractérise par rapport à la Néo-Zélandaise Blanche, par un plus fort niveau d'ovulation et inversement une plus faible aptitude à conserver les ovules fécondés.

L'évolution des valeurs des 3 types d'effets additifs entre la naissance et le sevrage, pour les effectifs par portée sevrée, traduit leurs effets sur la viabilité des lapereaux. Seule la Petite Russe a un effet génétique direct favorable sur la viabilité naissance-sevrage des lapereaux. Les lapereaux croisés Petite Russe qui ont un poids potentiel plus faible du fait de la plus petite taille adulte de la souche, n'auraient pas de facteur alimentaire limitant sous la mère. La petite taille adulte des mères expliquerait également l'effet maternel très défavorable de la Petite Russe sur le nombre de lapereaux sevrés par portée, du fait d'une plus faible aptitude laitière que dans les deux autres souches. L'opposition entre effets maternels (favorables) et grand-maternels (défavorables) de la Californienne sur le nombre de lapereaux sevrés par portée sevrée, pourrait avoir la même explication que celle émise depuis FALCONER (1955) et qui concernait la prolificité intra-souche chez la souris. Les lapines nées et ici élevées jusqu'au sevrage dans des portées plus nombreuses (effet maternel favorable de la Californienne sur la viabilité naissance-sevrage) auraient tendance, du fait de facteurs de milieu ne leur permettant pas d'extérioriser leur potentiel de croissance, à faire naître et élever elles-mêmes des portées d'effectifs plus faibles. Cette interprétation semble être confirmée ici par la présence d'un effet maternel non significatif de la Californienne sur le poids individuel moyen de lapereau sevré par portée, alors que cet effet maternel très significatif chez la Néo-Zélandaise Blanche se répercute par un effet grand-maternel très favorable de cette souche sur le nombre de lapereaux sevrés par portée sevrée. Les valeurs des effets maternels sur le poids individuel moyen de lapereau sevré par portée pourraient traduire l'aptitude laitière des lapines, plus élevée pour la Néo-Zélandaise Blanche que pour la Californienne et plus faible chez la Petite Russe, ce qui est toujours lié à sa plus petite taille.

Une plus forte aptitude laitière pourrait ainsi contribuer à expliquer l'effet favorable de l'ascendance maternelle Néo-Zélandaise Blanche sur la portée sevrée. Cette souche cumule des effets génétiques directs et maternels favorables sur le poids individuel moyen par portée des lapereaux sevrés. Pour ce caractère, l'opposition entre les 
2 souches Néo-Zélandaise Blanche et Petite Russe, entre effets maternels et grand-maternels, semble difficile à expliquer.

En ce qui concerne le poids total de portée, seuls les effets maternels sont importants à considérer. Ceux-ci reflètent les effets maternels sur le nombre de sevrés, confirmant que ce caractère conditionne principalement le poids total de portée, comme cela a été montré intra-souche par l'étude de la corrélation entre ces 2 caractères (RouvIER et al., 1973).

\section{B. Effets d'hétérosis}

Les gains cumulés par hétérosis direct et maternel moyens représentent 22 p. 100 pour les caractères de productivité numérique et pondérale par portée sevrée. Ils correspondent aux estimations du gain potentiel par hétérosis chez le porc, pour le poids de portée au sevrage, données par SELLIER (1976) et JOHNSON (1981), de 22 et 29 p. 100 respectivement. L'interprétation classique de l'hétérosis est la dominance orientée et la différence des fréquences géniques entre les souches croisées. L'existence d'une variation génétique de dominance pour le nombre de lapereaux sevrés et le poids de portée au sevrage a été montrée par Rollins et al. (1963) à l'intérieur d'une souche de lapins Néo-Zélandais Blancs.

L'hétérosis direct mis en évidence pour le nombre de nés vivants par mise bas et de sevrés par portée sevrée traduit une meilleure viabilité des produits croisés jusqu'au sevrage. Il intervient également sur le poids individuel moyen par portée des lapereaux sevrés, et corrélativement sur le poids total de portée sevrée. Les lapereaux croisés obtiendraient plus de lait maternel ou l'utiliseraient plus efficacement ainsi que l'aliment solide ingéré avant le sevrage.

L'hétérosis maternel est celui qui est intéressant à considérer pour l'amélioration de la performance de portée sevrée d'une lapine métisse. Il y a, sur le nombre de sevrés par portée sevrée, un maintien de l'hétérosis maternel moyen observé sur le nombre de nés vivants par mise bas. Ce dernier pourrait indiquer un contrôle génétique de dominance, du taux d'ovulation et des effets maternels sur la viabilité des embryons, ce qui rejoint les résultats de Hulot \& MATHERON (1979). Un effet d'hétérosis direct sur la production laitière pourrait expliquer que les lapines métisses sont capables d'élever un nombre supplémentaire de lapereaux par rapport aux lapines pures utilisées en croisement. L'hétérosis maternel est spécifique aux lapines métisses des couples de souches Californienne et Petite Russe, Néo-Zélandaise Blanche et Petite Russe. L'existence et l'importance pratique de l'hétérosis spécifique ont déjà été signalées par plusieurs auteurs dans différentes espèces de mammifères comme l'indique SELLIER (1982) dans une discussion sur la sélection pour l'utilisation en croisement.

\section{Choix du croisement pour l'amélioration de la productivité numérique et pondérale}

Les systèmes de croisement utilisés pour les espèces domestiques dépendent d'abord d'un paramètre biologique relatif au taux de reproduction qui détermine les possibilités de renouvellement des femelles des troupeaux. Dans l'espèce cunicole, les croisements de type rotatif qui permettent à l'éleveur de bénéficier des effets du croisement par l'introduction de mâles et d'auto-renouveler en continu ses femelles, étaient traditionnellement utilisées. Depuis 10 ans (Rouvier, 1981), se généralisent dans cette espèce à 
taux de reproduction élevé, des plans de croisement de type discontinu, à l'instar des autres espèces de mammifères polytoques (porcins) ou des espèces aviaires. L'objectif appliqué de cette recherche était de choisir une lapine croisée de forte productivité numérique et pondérale au sevrage à accoupler à un mâle de croisement terminal : la lapine croisée Californienne $\times$ Néo-Zélandaise Blanche a été sélectionnée : ses résultats sont nettement supérieurs à ceux de la lapine croisée réciproque et sa productivité pondérale en lapereaux de boucherie plus élevée que celle des lapines croisées Californienne $\times$ Petite Russe et Petite Russe $\times$ Néo-Zélandaise Blanche, qui ont également une forte productivité numérique, mais qui sont pénalisées sur le plan pondéral par leur ascendance Petite Russe.

La présente analyse permet d'expliquer comment interviennent la complémentarité et l'hétérosis entre ces 3 souches : la performance d'une lapine métisse Californienne $\times$ Néo-Zélandaise Blanche bénéficie, par complémentarité, d'une combinaison favorable d'effet maternel transmis par le mâle Californien, agissant sur l'ovulation et sur la viabilité naissance-sevrage et d'effet grand-maternel de la Néo-Zélandaise Blanche (sur la viabilité des lapereaux), sans hétérosis. Cette complémentarité apporte une amélioration de $18 \mathrm{p}$. 100 de la taille et du poids de portée au sevrage par rapport à la moyenne parentale. Par contre, les métisses Petite Russe $\times$ Néo-Zélandaise Blanche et Californienne $\times$ Petite Russe cumulent une combinaison d'effets additifs favorables et d'hétérosis maternels spécifiques. La complémentarité seule peut apporter une amélioration comparable à celle apportée par l'hétérosis maternel. Cette complémentarité ne s'exprime pas intra-souche compte-tenu d'une opposition entre effets maternels et grand-maternels dans le cas de la Californienne, des facteurs limitants de la Petite Russe liés à sa petite taille ou des effets maternels moyens de la Néo-Zélandaise Blanche sur les effectifs de lapereaux sevrés.

\section{Conclusion}

A notre connaissance, il n'existait pas chez le lapin d'interprétations des expériences de croisement de souches en termes d'effets de différentes origines génétiques en tenant compte des caractéristiques biologiques d'élaboration de la portée sevrée. Pour les caractères de la portée étudiés, les effets génétiques maternels et grand-maternels varient très significativement entre les 3 souches. Agissant parfois en opposition entre souches, notamment entre la Californienne et la Néo-Zélandaise Blanche, ils peuvent se combiner favorablement en croisement, expliquant une complémentarité entre effets génétiques additifs sur le même caractère. L'identification des effets additifs à des mécanismes biologiques (ovulation, viabilité des lapereaux, aptitude laitière des lapines) permet de caractériser les souches sur un plan biologique et de leurs effets génétiques sur les caractères étudiés. On pourrait donc prédire les effets de ces souches utilisées en croisement avec d'autres souches, comme mâles ou comme femelles. Cela indique l'intérêt de ce type d'expérience, pour une connaissance des souches, lorsque l'on peut la conduire sur les 2 générations successives de croisement. Une autre conclusion est que la complémentarité entre effets génétiques additifs seule ou l'hétérosis maternel, conduit à la supériorité d'une lapine métisse pour les caractères étudiés. Cela militerait en faveur de l'utilisation d'une sélection récurrente réciproque pour améliorer les caractères de portée des lapines métisses. A cet égard, il est important de connaître précisément les 
paramètres génétiques en pur et en croisement (héritabilités et corrélation génétique pur-croisement) pour discuter objectivement l'efficacité relative de cette méthode par rapport à la sélection classique intra-population.

Reçu le 25 février 1983.

Accepté le 7 février 1984.

\section{Remerciements}

Nous remercions les techniciens de l'Elevage expérimental de lapins de la Station d'Amélioration génétique des Animaux qui ont élevé les animaux et contrôlé leurs performances, ainsi que B. PouJARDIEU et les 2 lecteurs anonymes mandatés par la revue qui ont permis d'améliorer le manuscrit.

\section{Références bibliographiques}

Alenda R., Martin T.G., LASLey J.F., Ellersieck M.R., 1980. Estimation of genetic and maternal effects in crossbred cattle of Angus, Charolais and Hereford parentage. I. Birth and weaning weights. J. Anim. Sci., 50, 226-234.

Dickerson G., 1969. Experimental approaches in utilizing breed resources. Anim. Breed. Abstr., 37, 191-202.

EISEN E.J., Hörstgen-Schark G., SaXton A.M., Bandy T.R., 1983. Genetic interpretation and analysis of diallel crosses with animals. Theor. Appl. Genet., 65, 17-23.

FALCONER D.S., 1955. Patterns of response in selection experiments with mice. Cold Spring Harbor Symp. Quant. Biol., 20, 178-196.

Hulot F., Matheron G., 1979. Analyse des variations génétiques entre trois races de lapins de la taille de portée et de ses composantes biologiques en saillie post-partum. Ann. Génét. Sél. Anim., 11, 53-77.

Hulot F., Matheron G., 1981. Effets du génotype, de l'âge et de la saison sur les composantes de la reproduction chez la lapine. Ann. Génét. Sél. Anim., 13, 131-150.

Johnson R.K., 1981. Crossbreeding in swine : experimental results. J. Anim. Sci., 52, 906-923.

Matheron G., Poujardieu B., 1976. Hétérosis pour quelques caractères de reproduction chez le lapin. Analyse de plans de croisement. Bull. Tech. Dép. Génét. Anim. (Inst. Nat. Agron. Fr.), 24, 69-77.

Matheron G., Rouvier R., 1978. Etude de la variation génétique dans le croisement à double étage chez le lapin : performances de reproduction des lapines croisées et pures accouplées en croisement. $2^{e}$ Journée de la Recherche Cunicole en France, I.N.R.A.-I.T.A.V.I., Toulouse, 4-5 avril 1978, 5 pages ronéotypées.

Matheron G., MaulÉon P., 1979. Mise en évidence de l'action conjointe des effets directs, maternels et grand-maternels sur la taille de portée. Bull. Tech. Dép. Génét. Anim. (Inst. Nat. Rech. Agron. Fr.), 29-30, 232-274.

Rollins W.C., Casady R.B., Sittmann K., Sittmann D.B., 1963. Genetic variance component analysis of litter size and weaning weight of New-Zealand White rabbits. J. Anim. Sci., 22, 654-657.

Rouvier R., Poujardieu B., Vrillon J.L., 1973. Analyse statistique des performances d'élevage des lapines. Facteurs du milieu, corrélations, répétabilités. Ann. Génét. Sél. Anim., 5, 83-107.

ROUviER R., 1981. Les travaux de recherche français sur la sélection du lapin au cours des 10 dernières années (1970-1980). C.R. Acad. Agric., 67, 509-524.

Sellier P., 1976. The basis of crossbreeding in pigs. A review. Livest. Prod. Sci., 3, 203-226.

SELlier P., 1982. Selecting populations for use in crossbreeding. 2nd World Congress on Genetics applied to Livestock production. Madrid, 4-8 october 1982, 6, 15-49, Editorial Garsi, Madrid. 